The Baby Friendly Hospital Initiative

\author{
Kathryn Brackett
}

A Senior Thesis submitted in partial fulfillment of the requirements for graduation in the Honors Program Liberty University Spring 2014 
Acceptance of Senior Honors Thesis

This Senior Honors Thesis is accepted in partial fulfillment of the requirements for graduation from the Honors Program of Liberty University.

Kimberly Little, Ph.D.

Thesis Chair

Brianne Friberg, Ph.D.

Committee Member

Tracey Turner, M.S.N.

Committee Member

James Nutter, D.A.

Honors Program Director

Committee Member

Date 
Table of Contents

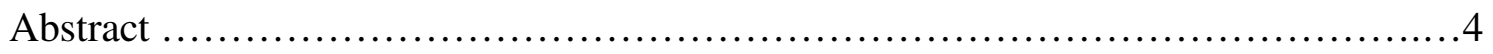

The Baby Friendly Hospital Initiative...............................................5

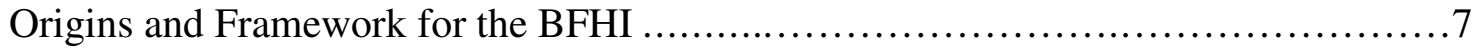

The Ten Steps to Successful Breastfeeding ...................................10

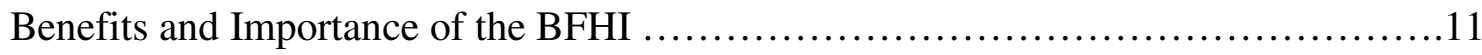

Health benefits of breastfeeding .............................................11

Breastfeeding as a public health issue ..........................................14

Implementation of the BFHI within the United States..............................14

Breastfeeding rates in the United States .....................................15

Status of BFHI implementation in the United States ............................17

Obstacles to the BFHI in the United States ....................................18

Benefits of widespread implementation of the BFHI in the United States.........23

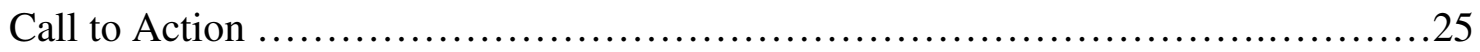

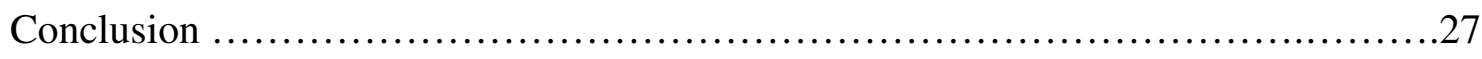

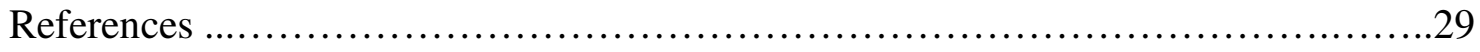




\begin{abstract}
The Baby-Friendly Hospital Initiative (BFHI) is a worldwide movement started by the World Health Organization (WHO) and the United Nations Children's Fund (UNICEF) that is aimed at providing hospitals with a certification that guarantees the best possible patient-centered care for mothers and infants. It focuses on breastfeeding as optimal infant nutrition, and implements strategies to encourage and support mothers to breastfeed their infants exclusively for six months after birth. The Ten Steps to Successful Breastfeeding are a set of 10 simple, easy to understand guidelines developed by WHO and UNICEF for implementing this initiative in the hospital setting and are used as the foundation for the BFHI. This initiative has been successful in many countries across the globe since its initiation in the early 1990s, but has yet to become widespread within the United States. This thesis will examine the background of this initiative, its benefits for mothers and infants, and evaluate the progress of its implementation within the United States.
\end{abstract}




\section{The Baby Friendly Hospital Initiative}

The Baby-Friendly Hospital Initiative was a movement launched in 1991 by WHO and UNICEF in order to protect and promote breastfeeding in hospitals all across the world. By the year 2009, over 20,000 hospitals in 156 countries had adopted this initiative and implemented exclusive breastfeeding practices in the hospital setting (WHO and UNICEF, 2009). Once a hospital has put this initiative into effect by going through a series of policy changes, they reach "Baby-Friendly" status. This thesis will be written as a literature review of the studies supporting this initiative, and will discuss the benefits and importance of breastfeeding and becoming a Baby-Friendly hospital, specifically within the United States.

Although culture and hospital policies are diverse across the globe, the BabyFriendly initiative remains "one of the greatest international health initiatives ever, uniting clinicians, researchers, and advocates around a simple set of guidelines, with the unique purpose of creating healthier human beings from day one" (Philipp, et al., 2001, p. 677). The benefits of early initiation of breastfeeding and continued breastfeeding up through the second year of life are innumerable, promoting healthier mothers, infants, and communities (Richman \& Cabrera, 2012). However, breastfeeding is difficult for a new mother to begin and sustain without proper support and education. According to research, less than one-third of American mothers who intended to breastfeed exclusively for three months after birth were successful (Gordon, 2012). Also, in 2011 it was reported that only $4 \%$ of United States hospitals provided the complete range of support for breastfeeding mothers (Center for Disease Control and Prevention, 2011). For these reasons, the Baby-Friendly hospital initiative seeks to implement early breastfeeding and 
provide support for mothers, providing the needed resources for them to breastfeed successfully.

Breastfeeding policies that are implemented in hospitals seeking Baby-Friendly status focus on exclusive breastfeeding within the postpartum period and place very high importance on patient education regarding the benefits of early and sustained breastfeeding. In a typical breastfeeding hospital policy, maternal education is an extremely significant part of the major recommendations (Philipp, 2010). These breastfeeding policies also place high importance on infant-parent bonding, and implement "rooming-in" in order to promote bonding and breastfeeding on demand (Hurst, 2013, p. 1).

Another important consideration within the Baby-Friendly hospital initiative is the practical application, and the attitudes of healthcare workers toward the implementation of the policy. In order for this policy to be launched and be successful in a hospital, the staff and healthcare workers (especially nurses) must be on board with the initiative. Educational interventions have been put in place to make healthcare professionals more competent within Baby-Friendly settings, allowing for better outcomes (Young, 2012).

The Baby-Friendly hospital initiative has major impacts globally and personally, benefitting infants and mothers, as well as healthcare professionals, hospitals, communities, and entire countries. Although the impact of this initiative has exhibited exceptional outcomes in many parts of the world, many in the United States are still uneducated on the benefits of the BFHI and lack the knowledge necessary to implement this policy. As of 2010, only $3 \%$ of hospitals in the United States were Baby-Friendly 
certified (Sadacharan, et al., 2012). Education about this policy and its importance in healthcare is essential to the implementation of Baby-Friendly policies in hospitals in the United States. This thesis will outline the 10 steps to successful breastfeeding and the breastfeeding policies put in place by Baby-Friendly hospitals, examine the status of breastfeeding implementation within the United States, review the potential benefits of this initiative in improving breastfeeding rates, as well as discuss the current status of the Baby-Friendly Hospital initiative within the United States, barriers to the initiative, and how this initiative is crucial to improving the health outcomes of Americans.

\section{Origins and Framework for the Baby-Friendly Hospital Initiative}

The Baby-Friendly Hospital Initiative (BFHI) was developed by WHO and UNICEF, and began its worldwide implementation in the early 1990s (WHO and UNICEF, 2009). This initiative gives hospitals and birthing facilities the resources and framework for promoting breastfeeding and helping mothers to achieve successful breastfeeding, through a series of policy changes. This policy is centered on the implementation of the Ten Steps to Successful Breastfeeding, each of which will later be discussed. These Ten Steps have a "timeless simplicity" that keeps the BFHI consistent worldwide, regardless of the obstacles that may stand in the way of making it an important policy in hospitals across the globe (Merewood, 2012). These Ten Steps transcend culture and difference in national policies, and due to its universality, the BFHI has become one of the most prominent health initiatives in the world. The BFHI brings together breastfeeding advocates, researchers and those in clinical practice through its set of simplistic and practical guidelines which aim to promote the health and well-being of all people, starting at birth (Merewood, 2012). This initiative is so distinct from other 
health movements in that discrepancy and disagreement are almost nonexistent.

Research repeatedly suggests that breastfeeding and mother-infant bonding not only have the best outcomes for infants and new mothers, but the best possible outcomes throughout the lifespan. The BFHI has been successful in increasing breastfeeding rates in every hospital where it is implemented, and so its promotion and growth must be encouraged in all parts of the world (Merewood, 2012).

The initiation of this policy was fueled by the Innocenti Declaration on the Protection, Promotion, and Support of Breastfeeding, a declaration made at a meeting of WHO/UNICEF policymakers at the Spedale degli Innocenti in Florence, Italy from July 30 to August 1 of 1990. This statement declared that, as a "global goal," all women should be provided with the resources and guidance to breastfeed exclusively from birth until four to six months of age, and continued breastfeeding thereafter with the introduction of complementary foods into the diet. In order to do this, the Innocenti Declaration calls for women to be empowered and for many societies to be challenged to become "breastfeeding cultures" instead of "bottle-feeding cultures." The Innocenti Declaration called every government, national authority and international authority to promote and encourage breastfeeding as best practice for all families (UNICEF and...Organization, 1990).

Some years after their original publication of the BFHI policy in 1991-1992, WHO and UNICEF networked with countries that adopted the initiative, and received feedback through a series of regional meetings with these countries (WHO and UNICEF, 2009). The recommendations and feedback from those adopting this initiative revealed that an updated policy was needed, with an actualization of the Global Criteria, and with 
special attention given to the new Global Strategy for Infant and Young Child Feeding, the HIV pandemic, and "mother-friendly care" (WHO and UNICEF, 2009, p. 4).

UNICEF evaluated these recommendations, and in collaboration with WHO, updated their policy in 2004-2005. Surveys and feedback from hospitals and clinicians actually using the BFHI were instrumental in providing the framework for the updated policy. BFHI experts worldwide reviewed these updates and conducted experimental studies in both developed and developing countries before publishing the first draft of the BFHI materials in 2006 (WHO and UNICEF, 2009). After trialing the new policy for one year, UNICEF and WHO used the results and feedback to fine-tune the policy, and a final draft of the policy was published in late 2007. Another update to these materials is expected to occur before the year 2018 (WHO and UNICEF, 2009).

The BFHI has grown exponentially since the first steps were taken in the 1990s, after the 1990 Innocenti Declaration on the Protection, Promotion, and Support of Breastfeeding (WHO and UNICEF, 2009). UNICEF and WHO's response to this declaration resulted in the mobilization of very few countries to adopt an initiative to promote breastfeeding. In 2009, after almost 15 years of work in promoting infant and young child nutrition, 156 hospitals had become involved with the BFHI and each claimed at least one "Baby-Friendly" designated hospital (WHO and UNICEF, 2009). The BFHI provides the necessary tools and resources for hospitals and birth centers to "enable" mothers, infants, and families to start life in the best way (WHO and UNICEF, 2009). The Global Strategy for infant and child nutrition from WHO and UNICEF places special emphasis on the importance of exclusive breastfeeding through six months of age, and continued breastfeeding up through the age of two years and beyond with approved 
complementary feeding (WHO and UNICEF, 2009).

\section{The Ten Steps to Successful Breastfeeding}

The core of the BFHI movement is centered on the Ten Steps to Successful Breastfeeding, developed by UNICEF and WHO. According to UNICEF and WHO, every maternity facility that provides services for newborn infants should:

1) Have a written breastfeeding policy that is routinely communicated to all health care staff, 2) Train all health care staff in skills necessary to implement this policy, 3) Inform all pregnant women about the benefits and management of breastfeeding, 4) Help mothers initiate breastfeeding within a half-hour of birth (which, as of 2009, is interpreted as placing babies in skin-to-skin contact with their mothers immediately after birth for at least an hour, and encourage mothers to recognize when their babies are ready to breastfeed and offer support if they need it), 5) Show mothers how to breastfeed, and how to maintain lactation even if they should be separated from their infants, 6) Give newborn infants no food or drink other than breast milk unless medically indicated, 7) Practise [sic] rooming in - allow mothers and infants to remain together - 24 hours, 8) Encourage breastfeeding on demand, 9) Give no artificial teats or pacifiers (also called dummies or soother) to breastfeeding infants, and 10) Foster the establishment of breastfeeding support groups and refer mothers to them on discharge from the hospital or clinic. (WHO and UNICEF1, 2009, p. 11)

In order to achieve Baby-Friendly status, facilities must first demonstrate adherence to these Ten Steps. 


\section{Benefits and Importance of the Baby-Friendly Hospital Initiative}

The benefits of the implementation of the BFHI are innumerable. The Innocenti Declaration, which is the aforementioned statement made in 1990 about the support of breastfeeding, said that "exclusive breastfeeding is the leading preventive child survival intervention" (WHO and UNICEF, 2009, p. 25). This statement claimed that through six months of exclusive breastfeeding and continued breastfeeding for two years or more, two million lives could be saved every year (WHO and UNICEF, 2009). Of course, the BFHI is a policy implemented for hospitals and birthing centers only, and this policy cannot be enforced upon mothers after they have left the hospital. However, according to WHO and UNICEF, research supports that mothers coming from hospitals that delay breastfeeding and provide supplemental nutrition for newborns are less likely to exclusively breastfeed upon leaving the facility (2009).

\section{Health Benefits of Breastfeeding}

Breastfeeding provides many benefits for both mothers and infants. WHO and UNICEF, in Section II of their 2009 BFHI publication, provide teaching sessions for maternity staff in facilities that decide to implement the BFHI. These education sessions focus heavily on the benefits of breast milk. According to these teachings, breast milk delivers nutrients and hydration that are incomparable to any other form of nutrition (WHO and UNICEF, 2009). When infants are breastfed exclusively (without other supplementation) and on demand (when mothers respond immediately to feeding cues), the water in breast milk is actually more than adequate to meet the baby's water requirements, even in hot, dry climates. Breast milk is unique for each mother and baby, adapting to the baby's gestational age, stage of lactation, and the time frame in which the 
baby is fed. According to WHO, UNICEF, and the American Academy of Pediatrics, for the first six months of a baby's life, breast milk is the only needed nutrition. After six months of age, breastfeeding should be continued and complementary foods added to the baby's diet. In addition to being high in nutrients and water content, breast milk provides immunity for the newborn child, giving him the antibodies he needs to fight infection. Without these antibodies passed from mother to child, the baby is poorly equipped to defend his body against infection (WHO and UNICEF, 2009).

The teaching in the BFHI curriculum also recounts that exclusive breastfeeding for six months decreases the risk of many common diseases such as diarrhea, respiratory infections, otitis media, allergies, and obesity. Apart from all these physical benefits, breastfeeding also has benefits developmentally and psychosocially. It allows for bonding between mother and child, making mothers more sensitive to cues from their babies. This leads to a loving relationship between mother and child, and babies tend to cry less when early skin-to-skin contact and breastfeeding are initiated and maintained. There seems to be a correlation between breastfeeding and IQ as well; one long-term study in Copenhagen by Mortenson, et al. showed that 27-year-olds who were breastfed as infants demonstrated significantly higher IQ scores (cited by WHO and UNICEF, 2009). Breastfeeding has also been shown to reduce the risk of chronic diseases later in life, such as diabetes, irritable bowel disease, high blood pressure, and high serum cholesterol (WHO and UNICEF, 2009).

A study conducted on the effect of early skin-to-skin contact also supports the BFHI (namely step four of the Ten Steps). This prospective cohort study published in 2010 by the California Perinatal Services Network looked at 21,842 mothers in 19 
hospitals who gave birth to one infant at full-term (37-40 weeks gestation). By taking into account the mother's feeding intentions prior to birth, her socio-demographic, intrapartum variables, and the duration of early skin-to-skin contact within the first three hours following birth, a correlation was found between these variables and breastfeeding exclusivity. There was a dose-response relationship between time spent in skin-to-skin contact and breastfeeding exclusivity: the longer the duration of early skin-to-skin contact between mother and newborn, the higher the likelihood of exclusive breastfeeding during the hospital stay (Bramson et al., 2010). The publishers of this study also pointed out that failure to breastfeed was usually due to hospital policies of separating mother and infant during the most crucial time of bonding. Within the first hour of birth, the infant is alert and able to latch on to the mother's breast without assistance. However, according to many hospital practices, the baby is taken away from the mother during this time in order to perform routine injections, cleaning and assessments. Loma Linda University Medical Center's Perinatal Services Network found that routine hospital practices such as infant assessment, suctioning, vitamin $\mathrm{K}$ injection, erythromycin application, swaddling, and mother-infant separation after birth impair maternal-infant bonding and breastfeeding practices. However, when the infant is placed in skin-to-skin contact with the mother immediately after birth (as is recommended by the BFHI and the Academy of Pediatrics), the infant is able to adjust better to life outside the womb, regulate his body temperature, conserve energy, and tends to have better nursing outcomes (Bramson et al., 2010). 


\section{Breastfeeding as a Public Health Issue}

In a 2012 publication on breastfeeding and human milk, the American Academy of Pediatrics (AAP) stated that in light of the innumerable and incomparable benefits of breastfeeding, "infant nutrition should be considered a public health issue and not only a lifestyle choice" (American Academy of Pediatrics, 2012, p. 827). In this publication, the AAP reiterated its recommendation of six months of exclusive breastfeeding after birth, and continued breastfeeding for at least a year with complementary foods introduced after six months. The AAP stated that all hospital initiatives to promote exclusive breastfeeding should be based on the Ten Steps by WHO and UNICEF. In the measurement of the quality of care provided by a medical facility, the rate of exclusive breastfeeding is a "crucial variable" (American Academy of Pediatrics, 2012, p. 835). In accordance with the BFHI objectives, the AAP strongly supports breastfeeding. The benefits of breastfeeding, nutritional and nonnutritional, provide the best health outcomes for mothers and infants, and promote good health across the lifespan. The risks associated with not breastfeeding are detrimental, and the AAP regards breastfeeding as a basic public health issue. The AAP strongly encourages pediatricians to support and advocate for healthy breastfeeding practices, and considers pediatricians as vital participants in helping to meet BFHI goals (American Academy of Pediatrics, 2012).

\section{Implementation of the Baby-Friendly Hospital Initiative within the United States}

The BFHI is expanding rapidly in the United States, due to a greater national understanding of the importance of breastfeeding, and how the maternal facility plays a vital role in encouraging lactation. More financial support has been given to this initiative, at the state and local levels, and the involvement of the Joint Commission and 
the Center for Disease Control and Prevention have encouraged the development of the BFHI within the United States (MacEnroe, 2010). However, according to several current studies and reports, the United States is still very far behind in implementing this initiative. Lack of education among maternity staff and the preference of convenience over health have put the United States at a deficit when it comes to breastfeeding and implementing the Ten Steps. As has been discussed, although the BFHI can only be implemented in the hospital or birth center setting, the ultimate goal of this initiative is to improve exclusive breastfeeding rates after discharge, by providing mothers with the necessary teaching, support, and materials to be successful at breastfeeding exclusively for six months.

\section{Breastfeeding Rates in the United States}

According to the Center for Disease Control and Prevention's (CDC) latest breastfeeding report from the year 2013, in light of the goals of the BFHI, the current breastfeeding rates in the United States are quite disheartening. Out of the entire population, $76.5 \%$ of mothers and babies ever breastfed, $37.7 \%$ were breastfeeding exclusively at three months, and only $16.4 \%$ of mothers and babies were exclusively breastfeeding at six months (National...Promotion, 2013). When compared to other countries implementing the BFHI, the United States is far behind. In 2011-2012, according to the Canadian Community Health Survey, the percentage of Canadian mothers who were breastfeeding exclusively at six months was 26\% (Statistics Canada, 2013). In less developed countries, between 2000 and 2011, rates of exclusive breastfeeding at six months were leaps and bounds above North America: $68 \%$ in Peru, 74\% in Cambodia, and 85\% in Rwanda (Save the Children, 2012). Industrialized 
countries, given their access to milk supplementation and the percentage of mothers in the workforce, usually have lower breastfeeding rates. However, since the rise of the BFHI, many industrialized countries have demonstrated excellent breastfeeding rates. Compared to the $49 \%$ of American mothers who are breastfeeding (with or without supplementation) at six months, $80 \%$ of Norwegian mothers, $72 \%$ of Swedish mothers, and $60 \%$ of Australian mothers are reported as continuing breastfeeding through six months (Save the Children, 2012).

The Healthy People 2010 goals for breastfeeding rates in the U.S. were as follows: $75 \%$ will ever breastfeed, $40 \%$ will be breastfeeding exclusively at three months, and $17 \%$ will be breastfeeding exclusively at six months (Bosco, 2007). According to the previous statistics given by the CDC for 2013, two of these three Healthy People 2010 goals have yet to be achieved. New Healthy People goals for the year 2020 have been published, which outline infant care and nutrition objectives to be accomplished in the next decade in the United States. In general, these objectives aim to "increase the proportion of infants who are breastfed" (National...Promotion, 2013, p. 6). Objective number 21 of Healthy People 2020 aims for the percentage of infants who have ever breastfed to increase from $76.5 \%$ to $81.9 \%$, and for the percentage of infants that are breastfed exclusively for six months to increase from $16.4 \%$ to $25.5 \%$. Objective number 22 is for an increase in the proportion of employers that have lactation support programs on-site, objective 23 to decrease the proportion of infants who receive supplemental formula within the first two days of life, and objective 24 to increase the percentage of live births that take place in facilities that give recommended care for breastfeeding mothers (National...Promotion, 2013). In order to achieve these goals, the BFHI must 
become known within the U.S., and the barriers to implementing the BFHI must be overcome in order to promote breastfeeding in all parts of the country.

\section{Status of BFHI Implementation in the United States}

In a 2012 group interview with representatives from several different countries, the current status of the BFHI in the United States was discussed with Trish MacEnroe, the director of Baby-Friendly USA. (Grguric, et al., 2012). MacEnroe reported that about $4 \%$ of hospitals within the United States were designated as Baby-Friendly. She reported that in 2012, about 350 facilities were in the process of becoming designated as BabyFriendly, and new funding through the CDC would probably bring at least 100 more facilities into the process of implementing the BFHI. As of 2012, the BFHI in the United States was focused on expanding the initiative within birth facilities, and had little activity within the community. The BFHI in the United States is run by Baby-Friendly USA, Inc., which is a non-profit organization that began in 1997 (Grguric, et al., 2012). Maternity experts are in charge of assessing each facility and sending a report to a panel that determines if they are compliant with the Baby-Friendly guidelines. In the 2012 interview, MacEnroe stated that the most important achievement for the BFHI in the United States up to that point was the recent increase in the number of participating facilities. The involvement of the CDC and all levels of the United States government was very beneficial for Baby-Friendly USA. MacEnroe said that some of the biggest challenges for the initiative in the United States were recognition of the importance of the BFHI, the lack of consistent and correct information about the initiative, and the lack of staff and financial resources. Because of this deficit, more public and private organizations have been giving grants to Baby Friendly USA (Grguric, et al., 2012). 
Technology has been the most important tool in spreading awareness of the initiative, and MacEnroe said in the interview that in the coming years, the BFHI in the U.S. would place their focus on developing their website and making more resources available through the use of technology (Grguric, et al., 2012).

Despite the progression of the BFHI in the United States within the past few years, movement toward "widespread" certification has been slow in comparison with other parts of the world (Sadacharan, et al., 2012, p. 359). Laura Haiek of Public Health Nutrition stated that the BFHI is an "effective strategy" to promote exclusive and maintained breastfeeding through six months of age. However, becoming compliant with the Ten Steps requires creating sufficient policy and revising maternal-infant service in order to make the mother and baby dyad the focus of care, and "many countries have been slow to implement it" (Haiek, 2012, p. 894).

\section{Obstacles to the BFHI in the United States}

A 2012 study published in the Journal of Human Lactation was focused on assessing the general knowledge of the BFHI among maternity staff in the United States. This simple study was conducted by calling 2,974 hospitals which represented all 50 states and the District of Columbia, and asking the maternity staff if their hospital was a “Baby-Friendly hospital” (Sadacharan, et al., 2012, p. 361). This study emulated the experience of an expecting mother who may call the hospitals in her area to look for a Baby-Friendly designated center. Of the 2,974 hospitals called, 2,851 of them were included in the final results of the study, due to 123 unresponsive or uncooperative hospitals. At the time, the Baby-Friendly USA website listed 3\% of the 2,851 hospitals as Baby-Friendly designated. However, according to the maternity staff respondents, 
$62 \%$ of the hospitals were Baby-Friendly, $23 \%$ were not Baby-Friendly, and $15 \%$ said they were unfamiliar with the term "Baby-Friendly" (Sadacharan, et al., 2012). In all, $75 \%$ of the respondents gave an incorrect answer to the surveyors. The study did however find a strong correlation between the number of Baby-Friendly designated hospitals in a state, and the number of correct responses given in that state. The BFHI has been active in the United States for over 15 years, but according to this study, the majority of maternity hospitals in the U.S. still do not understand the term "BabyFriendly." This study reveals the lack of knowledge among maternity staff members, and the need for widespread education about the BFHI in the U.S. (Sadacharan, et al., 2012). It is important to note however, that knowledge at the state level is correlated with the number of Baby-Friendly hospitals in that state.

In addition to the lack of knowledge about this initiative among maternity staff within the U.S., current maternity hospital practices are suboptimal when it comes to the support and implementation of breastfeeding. According to the CDC's National Survey of Maternity Practices in Infant Nutrition and Care, the average score of implementation of the Ten Steps in the majority of U.S. hospitals was about 65\% (American Academy of Pediatrics, 2012). Many hospitals (about 58\%) incorrectly instructed mothers to limit the time of each feeding, and $41 \%$ gave pacifiers to infants that were breastfed. In $30 \%$ of these birth centers, the majority of infants were given formula as supplementation, and $66 \%$ of the centers gave mothers free commercial formula upon discharge. In response to these statistics, the AAP states that there is "a need for major conceptual change in the organization of the hospital services for the mother and infant dyad" (American Academy of Pediatrics, 2012, p. 834). Maternity staff must be trained and educated about the 
benefits of exclusive breastfeeding and how to provide proper support and education for breastfeeding mothers. In doing so, they can get rid of long-held beliefs and attitudes about the sufficiency of formula feeding (American Academy of Pediatrics, 2012).

According to the CDC, the policies and practices of most U.S. hospitals are not congruent with international evidence-based recommendations for the best maternal care, and impede mothers' abilities to breastfeed (Center for...Prevention, 2011). This statement was given in response to a national survey in 2007-2009 of maternity hospitals, which showed that the majority of U.S. hospitals provided commercial formula to breastfed infants, only $14 \%$ of hospitals had a written breastfeeding policy, and less than one-third of hospitals provided support for breastfeeding mothers after discharge (Center for...Prevention, 2011).

In 2012, it was reported that only one-third of mothers who planned to breastfeed exclusively for at least three months were able to meet their goal (Gordon, 2012). The most prevalent factor in breastfeeding mothers' success was the implementation of formula feeding in the hospital after birth. Those who exclusively breastfed during their hospital stay were much more successful in exclusively breastfeeding in the months after discharge. According to the study, mothers who did not give any supplemental feedings to their babies during their hospital stay were "2.3 times more likely to achieve their breast-feeding intentions" (Gordon, 2012, p. 6). Although U.S. hospitals and birth centers can only influence maternal-infant care for a few days after birth before discharge, this influence has a profound impact on the success of breastfeeding long after discharge. Giving mothers and babies a consistent and supportive start with nutrition practices is extremely important for establishing exclusive breastfeeding after discharge. 
Without the support and encouragement of the maternal facility, mothers and babies are far less motivated to breastfeed. Breastfeeding is difficult, and when maternity staff cannot properly support mothers who want to breastfeed, or choose to offer easier, more convenient alternatives and supplementation, American mothers are very likely to give up on what is the best possible nutrition for their infant.

The attitudes of U.S. health care providers in implementing this initiative also interfere with the expansion and success of the BFHI in the United States. The BFHI designation process is difficult for many U.S. facilities because beliefs about breastfeeding hinder its success. In an evaluation of U.S. health professionals' insights regarding the BFHI, it was found that in general, health care providers expressed positive attitudes toward the BFHI (Chapman, 2012). They believe that the Baby-Friendly designation would make them more desirable as a maternal health care facility, and that it would promote consistency among staff as far as breastfeeding education. Correction to poor advice would not have to be made, because all staff would receive the same breastfeeding education, and all personnel would be able to give exemplary breastfeeding support (Chapman, 2012). The health professionals recognized breastfeeding as a worldwide initiative, having knowledge of the Ten Steps and understanding the importance of breastfeeding. There were, however, concerns about the actual implementation of the BFHI in their institutions. Some professionals felt that the implementation of Baby-Friendly practices would make their staff feel forced to accept the BFHI, make women feel pressured to breastfeed their infants, and take away the right for parents to make an "informed decision" to formula feed their babies (Chapman, 2012). The participants of this study also expressed reservations about the acceptance of 
the BFHI in their facilities, stating that many staff members are opposed to change, and that encouraging exclusive breastfeeding would require a lot more time spent in educating mothers, which is difficult due to the size of patient loads. Formula was identified as an easy "solution" to the time constraints of health care providers, and that staff is under a lot of pressure to discharge patients as soon as possible. The providers did state, however, that several of the Ten Steps were easy to implement, such as skin-toskin contact, rooming-in, and not supplying pacifiers to breastfeeding mothers (Chapman, 2012). Overall, this study showed the complexity of the BFHI for many U.S. hospitals and the barriers that must be overcome in order to implement the Ten Steps. However, as the publishers stated, the BFHI is achievable due to its incomparable advantages over current practice, and the Ten Steps are simplistic enough to give every facility the ability to achieve this initiative (Chapman, 2012).

The Jersey Shore University Medical Center (JSUMC) was the first hospital in New Jersey to seek Baby-Friendly status, and began the process in 2002 (McKeever \& St. Fleur, 2012). On its journey to Baby-Friendly implementation, this hospital encountered many obstacles in relation to the attitudes of health care professionals within the institution. Physician education was a challenge, as many physicians in the institution were unfamiliar with how to give proper breastfeeding education. Many physicians felt that if breastfeeding was actively promoted, mothers would feel guilty if they choose to formula-feed their infants (McKeever \& St. Fleur, 2012). Nurses at JSUMC were very hesitant to implement step four of the Ten Steps (skin-to-skin contact immediately after birth), because they were afraid the infants would become hypothermic if they were not placed under the warmer right after delivery. Nursing staff at JSUMC also believed that 
mother-baby separation in postpartum care promoted healing and rest for the mother;

however, this practice has not shown better outcomes for the mother or infant, and can actually impede breastfeeding success (McKeever \& St. Fleur, 2012). Therefore, the implementation of step seven (24-hour rooming-in) was also difficult for staff at JSUMC, and they needed education in order to dispel "the perceived need" for mother-infant separation (McKeever \& St. Fleur, 2012, p. 313).

JSUMC used education and patient satisfaction surveys to help their staff to realize that Baby-Friendly practices would be beneficial for their patients, as well as their institution. They reported that the journey to Baby-Friendly status was a challenge due to cultural norms within the United States, and they needed to change staff attitudes in order to transition from traditional care to evidence-based practice (McKeever \& St. Fleur, 2012).

\section{Benefits of Widespread Implementation of the BFHI in the United States}

In spite of reservations about implementing the BFHI in the United States, the success of this initiative would result in not only better outcomes for mothers, infants and families, but reduced medical costs due to the decrease in chronic diseases which are correlated with not breastfeeding. As outlined in the previous section about the benefits of breastfeeding, diseases such as otitis media, gastrointestinal disease, lower respiratory tract infections, obesity, and type I diabetes can all be prevented through exclusive breastfeeding for six months. One study, published in 2010, examined national spending on each of these diseases along with necrotizing enterocolitis, atopic dermatitis, sudden infant death syndrome, childhood asthma, and childhood leukemia (each of which can also be associated with suboptimal breastfeeding practices) to predict national savings if 
80-90\% of U.S. mothers breastfed exclusively for six months (Bartick, et al., 2010). The results of this study indicated that if $90 \%$ of families in the United States could comply with the recommendation to breastfeed exclusively for six months, the U.S. would save $\$ 13$ billion a year in medical costs, and prevent over 911 deaths, which are conservative estimates (Bartick, et al., 2010). These results indicate that the breastfeeding rates in the U.S. are suboptimal, that it would be cost-effective to invest in a program to promote breastfeeding according to evidence-based recommendations, and that a "national coordinated effort" to increase breastfeeding rates is needed (Bartick, et al., 2010).

In a statement published in the 2009 BFHI policy, Innocenti said that recent scientific evidence and experience have put health professionals in a prime position to implement the BFHI (WHO and UNICEF, 2009). They said, however, that most professionals in the field have not been sufficiently trained to implement all of this knowledge. All of the materials and guidelines are available to health care professionals, and putting this initiative into practice is a matter of urgency. To reiterate a statement by the executive officers at WHO and UNICEF, Innocenti said, "There can be no delay in applying the accumulated knowledge and experience to help make our world a truly fit environment where all children can thrive and achieve their full potential" (WHO and UNICEF, 2009, p. 26). As has been evidenced in previous literature reviews, these statements made in 2009 are still true for the Baby-Friendly movement in the United States. Most maternity facilities are still deficient in the knowledge and implementation of the BFHI, and this is due to a lack of education.

In a study conducted by the Association of Women's Health, a pretest-posttest quasi-experimental design was used to evaluate 40 health care professionals 
(pediatricians, obstetricians, and nurses) and their understanding of maternity care

practices in accordance with the BFHI (Young, 2012). The objective of this study was to see if education about the BFHI would increase knowledge of maternity care practices and result in more positive attitudes toward the initiative. A pretest was administered, then education about BFHI practices was given, and then a posttest was given to evaluate change in knowledge and attitude. On the survey of 10 knowledge questions, the average pretest score was $76 \%$, which increased to $95 \%$ after the training. Likert scale questions were also given in order to evaluate attitude regarding the BFHI, with a score of 1 being strongly disagree and a score of 5 being strongly agree. The average score on the pretest was 3.84 , and the average posttest score was 4.53 , showing "a strongly significant increase in positive attitude toward Baby-Friendly Hospital Initiative maternity care practices" (Young, 2012, p. 159). This study is evidence that education regarding the BFHI has positive outcomes, and that maternity staff is adaptable and capable of changing their attitudes regarding this initiative.

\section{Call to Action}

In 2011, the U.S. Surgeon General published a Call to Action to Support Breastfeeding, which has been an important resource for Baby-Friendly USA in implementing the BFHI. In the publication, the Surgeon General identified several barriers to breastfeeding in the United States. Not only are U.S. women unaware of the incomparable benefits of breastfeeding, but many health professionals believe that the new innovations in commercialized infant formula make it equivalent to human milk (U.S. Department... Services, 2011). Also, the lack of breastfeeding education for expecting mothers results in unrealistic expectations about breastfeeding and uncertainty 
about breastfeeding techniques. Because breastfeeding is considered the natural way to feed a baby, many mothers expect the practice to be easy, and give up when they find that it is difficult. Also, breastfeeding is often viewed by Americans as inconvenient, requiring mothers to sacrifice their time and freedom (U.S. Department... Services, 2011). The Surgeon General states that current practices for education about breastfeeding are not sufficient; many mothers end up relying solely on written information to teach them about breastfeeding, when they should be receiving hands-on teaching and support from health care professionals.

Another barrier identified by the Surgeon General is the lack of family and social support for breastfeeding. Those who are surrounded by proponents of breastfeeding are more likely to be successful, whereas those who are influenced by negative attitudes regarding breastfeeding are not as successful (U.S. Department...Services, 2011). Another barrier to breastfeeding that is specific to U.S. culture is the disapproval of breastfeeding in public. In American culture, breasts are seen as sexual objects, and so breastfeeding in public is seen as extremely uncomfortable. Many breastfeeding mothers feel embarrassment and do not breastfeed in public for fear of being stigmatized, and this has been cited as a reason for mothers to use supplemental formula or stop breastfeeding (U.S. Department...Services, 2011). Problems with breastfeeding such as pain, leaking milk, mastitis, difficulty with infant latching and fear of insufficient milk supply have also been cited reasons for mothers to stop breastfeeding. Even when seeking professional help for these issues, many women have trouble in receiving consistent information from their healthcare providers (U.S. Department...Services, 2011).

The barrier that is most relevant to this discussion about the BFHI is the 
insufficiency of maternal facilities in providing adequate breastfeeding support:

Studies have identified major deficits relevant to breastfeeding in hospital policies and clinical practices, including a low priority given to support for breastfeeding and education about it, inappropriate routines and provision of care, fragmented care, and inadequate hospital facilities for women who are breastfeeding. (U.S. Department...Services, 2011, p. 15)

According to the Surgeon General, most health care professionals do not realize the influence that they have on a mother's decision to breastfeed. Many professionals feel that they do not have the knowledge base or confidence to assist mothers with breastfeeding, and this ambivalence contributes greatly to the impaired success of mothers who desire to breastfeed (U.S. Department...Services, 2011).

In response to the current breastfeeding statistics and these barriers in U.S., the Surgeon General voices a Call to Action. The BFHI is supported and recommended as an important resource for hospitals to use to collaborate and make maternity care practices fully supportive of breastfeeding in the United States. The Surgeon General recommends that implementation of the BFHI be accelerated and incentives for the initiative be created in order to promote consistency and accuracy in hospital breastfeeding practices (U.S. Department...Services, 2011).

\section{Conclusion}

In conclusion, implementing the BFHI is a necessary step in providing the best evidence-based care for mothers and infants in the healthcare setting. Breastfeeding provides countless benefits for mothers and infants, and prevents a variety of diseases, making it the best choice in nutrition for every mother and infant dyad. Several countries 
around the world have implemented this initiative and been highly successful,

demonstrating increases in breastfeeding rates and better long-term health outcomes. The resources and guidelines set in place by WHO and UNICEF, namely the Ten Steps to Successful Breastfeeding, transcend culture and provide the necessary tools for health care facilities to develop a breastfeeding policy that will guarantee better outcomes for mothers, infants, and families. Although the United States has been involved with this initiative for over 15 years, breastfeeding rates are still suboptimal and health care facilities are still unaware of the BFHI. Due to barriers in education, understanding, and cultural constructs, the United States has been largely unsuccessful in implementing this initiative. However, with the promotion of this initiative and the education of health care workers and families, the BFHI has the potential to revolutionize maternal-child care in the United States. Education about the BFHI leads health care professionals to change their attitudes and biases about the initiative, and opens doors to implementing the BFHI in their facilities. Opportunities to advance the BFHI in the United States are more abundant now than ever. If the entire health care community within the United States would embrace and implement this initiative, it would not only benefit the health of mothers and infants, but greatly benefit the country as a whole. 


\section{References}

American Academy of Pediatrics (2012). Breastfeeding and the use of human milk.

Pediatrics, 3(129), e827-e842. doi: 10.1542/peds.2011-3552

Ammeson, J. (2011). Nurses at forefront of Baby-Friendly hospital initiative. Hoffman Estates, IL: Gannett Healthcare Group.

Bartick, M. \& Reinhold, A. (2010). The burden of suboptimal breastfeeding in the United States: A pediatric cost analysis. Pediatrics, 125, e1048. doi: 10.1542/peds.20091616

Bosco, M.L. (2007). Healthy People 2010 breastfeeding objectives revised. North Andover, MA: Fitzgerald Health Education Associates, Inc.

Bramson, L., Lee, J.W., Moore, E., Montgomery, S., Neish, C., Bahjri, K., \& Melcher, C.L. (2010). Breastfeeding during the maternity hospital stay infant contact during the first 3 hours following birth on exclusive: Effect of early skin-to-skin mother. Journal of Human Lactation, 26(130), 130-134.

doi:10.1177/0890334409355779

Center for Disease Control and Prevention (2011). Many hospitals do not fully support breastfeeding. HealthDay News. Ipswich, MA: Health News.

Chapman, D.J. (2012). Health professionals' insights regarding the Baby-Friendly Hospital Initiative. Journal of Human Lactation, 28(3), 315. doi: 10.1177/0890334412452870. 
Gordon, S. (2012). Only a third of U.S. moms who plan to breast-feed meet goals.

HealthDay News. Retrieved from http://consumer.healthday.com/women-shealth-information-34/breast-feeding-news-82/only-a-third-of-u-s-moms-whoplan-to-breast-feed-meet-goals-665305.html

Grguric, J., Wen, R.A., Kylberg, E., Ashmore, S., \& MacEnroe, T. (2012). International perspectives on the Baby-Friendly initiative. Journal of Human Lactation, 28, 281.

Haiek, L. (2012). Measuring compliance with the Baby-Friendly hospital initiative. Public Health Nutrition, 15(5), 894-905.

MacEnroe, T. (2010). The Baby-Friendly hospital initiative. Breastfeeding Medicine 5(5), 247. doi: $10.1089 / \mathrm{bfm} .2010 .0035$

McKeever, J., \& St. Fleur, R. (2012). Overcoming barriers to Baby-Friendly status: One hospital's experience. The Journal of Human Lactation, 28(3), 312-314. doi: $10.1177 / 0890334412440627$

Merewood, A. (2012). WHO/UNICEF's Baby-Friendly initiative: The big picture. Journal of Human Lactation, 28(3), 271. doi: 10.1177/0890334412447788

National Center for Chronic Disease Prevention and Health Promotion (2013). Breastfeeding report card 2013. Atlanta, GA: Centers for Disease Control and Prevention.

Philipp, B. L., Merewood, A., Miller, L. W., Chawla, N., Murphy-Smith, M. M., Gomes, J. S., \& ... Cook, J. T. (2001). Baby-Friendly hospital initiative improves breastfeeding initiation rates in a US hospital setting. Pediatrics, 108(3), 677. 
Philipp, B.L. (2010). ABM clinical protocol \#7: model breastfeeding policy (revision 2010). Breastfeed Med 2010, 5(4), 173-7. Academy of Breastfeeding Medicine Protocol Committee.

Registered Nurses Association of Ontario (2003). Breastfeeding best practice guidelines for nurses. Toronto, Canada: Registered Nurses Association of Ontario.

Richman, S., \& Cabrera, G. (2012). Breastfeeding: Global trends and practices. Glendale, CA: Nursing Practice Council.

Sadacharan, R., Santana, S., Sanchez, E., Matlak, S., Grossman, X., Makrigiorgos, G., \& Merewood, A. (2012). Are you Baby-Friendly? Knowledge deficit among US maternity staff. Journal of Human Lactation, 28(359), 359-361. doi:10.1177/0890334412437039.

Save the Children (2012). State of the world's mothers 2012. Save the Children International: London. Retrieved from http://www.savethechildren.ca/document.doc?id=195

Statistics Canada (2013). Study: Breastfeeding trends in Canada, 2003 and 2011/2012. The Daily. Retrieved from http://www.statcan.gc.ca/dailyquotidien/131125/dq131125c-eng.htm

UNICEF (2013). The baby-friendly hospital initiative. Retrieved from http://www.unicef.org/programme/breastfeeding/baby.htm

UNICEF and The World Health Organization (2012). The baby-friendly initiative. Retrieved from http://www.babyfriendlyusa.org/about-us/baby-friendly-hospitalinitiative 
UNICEF and The World Health Organization (1990). Innocenti declaration on the protection, promotion and support of breastfeeding. Florence, Italy, WHO/UNICEF (Spedale degli Innocenti).

U.S. Department of Health and Human Services (2011). The Surgeon General's call to action to support breastfeeding. U.S. Department of Health and Human Services, Office of the Surgeon General: Washington, D.C. Retrieved from http://www.surgeongeneral.gov/library/calls/breastfeeding/calltoactiontosupportbr eastfeeding.pdf

World Health Organization (1989). The ten steps to successful breastfeeding, protecting, promoting and supporting breast-feeding: The special role of maternity services. Geneva, Switzerland: World Health Organization.

WHO and UNICEF (2009). Baby-friendly hospital initiative: Revised, updated and expanded for integrated care. Geneva, Switzerland: World Health Organization.

Young, M.H. (2012). Changing knowledge and attitude toward maternity care practices consistent with the Baby-Friendly Hospital initiative. Association of Women's Health, Obstetric and Neonatal Nurses: Proceedings of the 2012 AWHONN Convention, S159. 